\title{
A New Item-Based Collaborative Filtering Algorithm to Improve the Accuracy of Prediction in Sparse Data
}

\author{
Wentao Zhao ${ }^{1} \cdot$ Huanhuan Tian ${ }^{1} \cdot$ Yan $\mathrm{Wu}^{1} \cdot$ Ziheng Cui $^{1} \cdot$ Tingting Feng $^{1}$
}

Received: 25 October 2021 / Accepted: 4 February 2022

(c) The Author(s) 2022

\begin{abstract}
In memory-based collaborative filtering (CF) algorithms, the similarity and prediction method have a significant impact on the recommendation results. Most of the existing recommendation techniques have improved different similarity measures to alleviate inaccurate similarity results in sparse data, however, ignored the impact of sparse data on prediction results. To enhance the adaptability to sparse data, we propose a new item-based CF algorithm, which consists of the item similarity measure based vague sets and item-based prediction method with the new neighbor selection strategy. First, in the stage of similarity calculation, the Kullback-Leibler (KL) divergence based on vague sets is proposed from the perspective of user preference probability to measure item similarity. Following this, the impact of rating quantity is further considered to improve the accuracy of similarity results. Next, in the prediction stage, we relax the limit of depending on explicitly ratings and integrate more rating information to adjust prediction results. Experimental results on benchmark data sets show that, compared with other representative algorithms, our algorithm has better prediction and recommendation quality, and effectively alleviates the data sparseness problem.
\end{abstract}

Keywords Recommendation systems $\cdot$ Collaborative filtering $\cdot$ Sparse data $\cdot$ Vague sets $\cdot$ Item similarity $\cdot$ Prediction

$\begin{array}{ll}\text { List of Abbreviations } \\ \text { CF } & \text { Collaborative filtering } \\ \text { KL } & \text { Kullback-Leibler } \\ \text { SPCC } & \text { Sigmoid and Pearson correlation coefficient } \\ \text { ACOS } & \text { Adjusted cosine similarity } \\ \text { JMSD } & \text { Jaccard and mean squared differences } \\ \text { NHSM } & \text { New heuristic similarity model } \\ \text { OS } & \text { Our developed similarity } \\ \text { BCF } & \text { Bhattacharyya coefficient in CF } \\ \text { KLCF } & \text { KL-based similarity measure } \\ \text { RPA } & \text { A recursive prediction algorithm } \\ \text { IRP } & \text { An iterative rating prediction algorithm } \\ \text { COS } & \text { Cosine similarity } \\ \text { PCC } & \text { Pearson correlation coefficient } \\ \text { MSD } & \text { Mean absolute difference } \\ \text { WA } & \text { Weighted average } \\ \text { MC } & \text { Mean centering } \\ \text { RJaccard } & \text { Related Jaccard } \\ \text { RJMSD } & \text { Related Jaccard and Mean squared differences }\end{array}$

Yan $\mathrm{Wu}$

2537735476@qq.com

1 School of Computer Science and Technology, Henan Polytechnic University, Jiaozuo 454003, China

$\begin{array}{ll}\text { URP } & \text { A new user rating prediction } \\ \text { INH-BP } & \text { A new adaptable prediction approach } \\ \text { ML-100K } & \text { MovieLens100K } \\ \text { ML-1M } & \text { MovieLens1M } \\ \text { MAE } & \text { Mean absolute error } \\ \text { RMSE } & \text { Root Mean square error } \\ \text { RSP } & \text { The rate of successful predictions } \\ \text { RPP } & \text { The rate of perfect predictions }\end{array}$

\section{Introduction}

With the advent of the big data era, the scale of information has expanded significantly. Especially in the field of e-commerce, users must devote more time and energies to finding valuable information, which makes the information overload problem [26] prominent. Recommendation system [23] is the main information filtering technology, which has been widely used in various online business fields such as video, music, e-commerce, advertising, news, etc. Recommendation algorithms are typically divided into three categories: collaborative filtering, content-based, or a hybrid of these two approaches. Collaborative filtering (CF) $[17,35]$ analyzes user preferences based on explicit data, and helps users 
obtain the content that they are interested in quickly and accurately, provide personalized need. In this research, the item-based CF algorithm is chosen for further improvement due to its simple and efficient. The recommendation process of item-based $\mathrm{CF}$ algorithm mainly involves two phases. The first phase is to search the nearest neighbors of the target item according to similarity values. The second phase is to predict user preferences based on the rating information of the nearest neighbors. Therefore, the design of similarity and prediction formulas has a crucial impact on recommendation results. However, as the number of users and items increases, cold start and data sparse problems are extremely severe, resulting in the quality of selected neighbors and the ability of accurate predictions are seriously affected.

In the stage of computing similarity values, some representative similarity measures, mainly including Sigmoid and Pearson Correlation Coefficient (SPCC) [16], Adjusted Cosine Similarity (ACOS) [25], Jaccard and Mean Squared Differences (JMSD) [4], New Heuristic Similarity Model (NHSM) [15], and Our developed Similarity (OS) [13], have been designed. They only rely on co-rated cases and can quickly return recommendation results. However, in extremely sparse data, the accuracy of similarity results is seriously affected. To address the limitation of co-rated cases, some state-of-the-art similarity measures, such as Bhattacharyya Coefficient in CF (BCF) [21] and KL-based Similarity Measure (KLCF) [28] have been presented based on the probability distribution of ratings. They maximally utilize all rating information to generate more accurate similarity results, which alleviates the problems of sparse data and cold start. However, these two measures add the corresponding item similarity when calculating user similarity, which involves massive Cartesian calculation, resulting in lower computation efficiency.

In the conventional item-based prediction methods, if a given user rates the nearest neighbors of the target item, then these information will be aggregated to predict unknown ratings. Otherwise, these potentially valuable contributions will be filtered out. This may aggravate the problem of sparse data in turn. To address this problem, some advanced prediction methods, a Recursive Prediction Algorithm (RPA) [36] and an Iterative Rating Prediction algorithm (IRP) [39], have been presented. They enable more ratings to participate the prediction process, to improve the accuracy of prediction. However, the related parameters have to be adjusted to determine the optimal value.

Based on the above analysis, due to cold start $[6,31]$ and sparsity issues [20,38], the quality of selected neighbors and the ability of accurate predictions are seriously affected. To alleviate these problems, we propose a new item-based CF model in this paper, which consists of an improved similarity measure and prediction method. Our contributions are mainly summarized as follows:
- The KL divergence based on vague sets is proposed from the perspective of user preference probability to measure item similarity. It relaxes the constraint of co-rated cases, and possesses good flexibility in sparse data. Meanwhile, only item similarity is required to calculate, which makes a high computational efficiency.

- The weight factor $\alpha$ is defined to emphasize the number of ratings in the proposed similarity measure. It can further improve the accuracy of similarity results in extremely sparse data.

- A item-based prediction method with a new neighbor selection strategy is presented. It allows more neighbors to participate in the prediction process, which effectively enhance the accuracy of prediction. What's more, it does not involve parameter selection and is easy to implement.

The rest parts of this study are organized as follows. The related work is briefly introduced regarding the existing item-based similarity measures and prediction methods in Sect. 2. Then, a new item-based CF algorithm is proposed in details in Sect. 3. Experimental results on benchmark data sets are described and discussed in Sect. 4. Finally, the conclusion and future work are given in Sect. 5.

\section{Related Work}

In this section, we briefly review the similarity and prediction work related to the methods of this study in Sects. 2.1 and 2.2 , respectively.

\subsection{Item-Based Similarity Measures}

In general, the traditional similarity measures are mainly divided into three categories, namely numerical, structural, and hybrid similarity. Numerical similarity calculates users or items similarity according to rating values. Cosine Similarity (COS) [7] takes the historical ratings on each item as a vector, and the similarity result is expressed by the cosine value of two vectors. The formula is defined in Eq. (1). Pearson Correlation Coefficient (PCC) [9] calculates similarity results by measuring the linear relationship between items on co-rated users, which is shown in Eq. (2). Besides, Mean Absolute Difference (MSD) [24] is computed by the average deviation of any two item ratings. The formula is expressed in Eq. (3):

$$
\operatorname{sim}(i, j)^{\operatorname{COS}}=\frac{\sum_{u \in U_{i} \cap U_{j}} r_{u i} \cdot r_{v i}}{\sqrt{\sum_{u \in U_{i} \cap U_{j}} r_{u i}^{2}} \cdot \sqrt{\sum_{u \in U_{i} \cap U_{j}} r_{v i}^{2}}}
$$


$\operatorname{sim}(i, j)^{\mathrm{PCC}}=\frac{\sum_{u \in U_{i} \cap U_{j}}\left(r_{u i}-\bar{r}_{i}\right) \cdot\left(r_{v i}-\bar{r}_{j}\right)}{\sqrt{\sum_{u \in U_{i} \cap U_{j}}\left(r_{u i}-\bar{r}_{i}\right)^{2}} \cdot \sqrt{\sum_{u \in U_{i} \cap U_{j}}\left(r_{v i}-\bar{r}_{j}\right)^{2}}}$

$\operatorname{sim}(i, j)^{M S D}=1-\frac{\sum_{u \in U_{i} \cap U_{j}}\left(r_{u i}-r_{u j}\right)^{2}}{\left|U_{i} \cap U_{j}\right|}$

where $\bar{r}_{i}$ is the average values of item $i . r_{u i}$ is the rating that user $u$ rates on item $i . U_{i}$ represents the sets of users rated on item $i$.

Structural similarity is different from numerical similarity, which focuses on whether the rating values exist or not. As one of the classic structural similarities, Jaccard [18] only emphasizes the rate of co-rated cases. The formula is shown in Eq. (4):

$\operatorname{sim}(i, j)^{\mathrm{Jaccard}}=\frac{\left|U_{i} \cap U_{j}\right|}{\left|U_{i} \cup U_{j}\right|}$

Hybrid similarity comprehensively considers the numerical and structural information of user ratings. It can make up for the shortcomings of the first two types of similarity and effectively enhance the accuracy of similarity calculation. JMSD is a combination of Jaccard and MSD, and SPCC integrates Sigmoid function and PCC, which have better prediction quality. Their expressions are the following, respectively:

$\operatorname{sim}(i, j)^{\mathrm{JMSD}}=\operatorname{sim}(i, j)^{\mathrm{Jaccard}} \cdot \operatorname{sim}(i, j)^{\mathrm{MSD}}$

$\operatorname{sim}(i, j)^{\mathrm{SPCC}}=\operatorname{sim}(i, j)^{\mathrm{PCC}} \cdot \frac{1}{1+\exp \left(-\frac{\left|U_{i} \cap U_{j}\right|}{2}\right)}$

However, these typical similarity measures all heavily rely on co-rated users. With the rapid expansion of users and items, the data set becomes extremely sparse, resulting in unreasonable similarity results or even nonexistent.

At present, some similarity measures based on heuristic strategy have been proposed. Ahn et al. [1] presented a similarity measure named PIP using the specific domain meaning, which was consisted of the product of three components (the proximity, the impact, and the popularity). To simplify and normalize PIP model, Liu et al. [15] presented NHSM, combining the rate of co-rated cases and user rating preferences. These measures can better distinguish similarity values between users or items even in sparse data. However, they are only effective when users have co-rated cases. To address this constraint, from the perspective of probability distribution, a linear similarity BCF [21] based on Bhattacharyya coefficient and a hybrid similarity KLCF [28] based on KL divergence were presented, respectively. These two schemes can apply all rating information rated by a pair of users to comprehensively calculate similarity values. In addition, Wang et al. [30] introduced $\alpha$-divergence to calculate item similarity, and further added the influence of the proportion of co-rated cases. To achieve simple and efficient recommendation, Bag et al. [3] proposed a new structural similarity named RJaccard, which not only considered co-rated cases, but also emphasized the importance of the number of non co-rated cases. Similarly, Gazdar et al. [13] proposed a new similarity measure OS, which comprehensively utilized the proportion of non common ratings and relative absolute rating difference. Wang et al. [29] proposed a general framework by combining similarity measurement to improve the prediction accuracy with less memory and time. To sum up, many similarity measures have been presented from different perspectives to enhance the accuracy of similarity results. However, this is still a need for further research.

\subsection{Item-Based Prediction Methods}

The two classic prediction methods, Weighted Average (WA) [5, 14] and Mean Centering (MC) [32], have been widely applied to generate prediction. WA took into account similarity values between items and the ratings given by nearest neighbors. MC added further the average values of items to adjust prediction results. Among them, the number of neighbors had significant influence on prediction results. Their formulas are expressed as follows:

$p_{u i}=\frac{\sum_{j \in N(i)} \operatorname{sim}(i, j) \cdot r_{u j}}{\sum_{j \in N(i)}|\operatorname{sim}(i, j)|}$

$p_{u i}=\bar{r}_{i}+\frac{\sum_{j \in N(i)} \operatorname{sim}(i, j) \cdot\left(r_{u j}-\bar{r}_{j}\right)}{\sum_{j \in N(i)}|\operatorname{sim}(i, j)|}$

where $p_{u i}$ is the predicted rating obtained from the prediction formula. The similarity value between $i$ and $j$ is $\operatorname{sim}(i, j)$.

In addition to the fundamental prediction methods, many researchers have put forward improvement strategies from different perspectives. Singh et al. [27] presented a predictive approach called $Z$-Score, which considered the rating differences by converting the ratings to $\mathrm{z}$-scores and calculating the weighted average of $z$-scores. A new User Rating Prediction (URP) [19] algorithm was proposed to predict ratings for items, which assumed that similar users may be interested in similar items. Alhijawi et al. [2] introduced a new adaptable prediction approach (INH-BP). It customized 
predictors for each active user to suit the user environment, thereby improving the accuracy of prediction. However, in the case of sparse data, these conventional prediction methods utilize neighbor items only from these who have already been rated by the given user, leading to poor prediction performance. To ease the problem, RPA [36] has been proposed, which enables a larger proportion of nearest neighbors to be included in the prediction process. Recently, based on RPA, IRP [39] has been further proposed. It sufficiently exploits ratings rated by direct and indirect neighbors to iteratively update the predicted ratings matrix. However, it is required to set a proper iterative parameter to achieve a stable state, which makes a long running time. In summary, it is still worth further introducing item-based prediction methods, to improve the accuracy of predictions in sparse data.

\section{The Proposed Model}

In this section, we aim to construct a CF model (ISP) that fuses the new item similarity with prediction method. First, The KL divergence based on vague sets is introduced to measure item similarity. Then, the impact of rating quantity is further considered in our similarity measure. In addition, we propose an improved prediction method with the new neighbor selection strategy.

\subsection{The Item Similarity Measure Based on Vague Sets}

\subsubsection{Definitions of Fuzzy Sets and Vague Sets on Recommendation Systems}

When people make judgments or decisions on vague and complex objective issues, they are often uncertain. The theory of fuzzy sets [33] was first put forward to declare the information of uncertain decision-making. Likewise, in recommendation systems, user's decision-making on items is often vague and no sharp class boundaries. Therefore, based on this association between fuzzy sets and recommendation systems, we first define a fuzzy set $A$, which represents overall preference of all users who have rated the given item $i$.

Definition 1 (Fuzzy sets) Let $X$ donate the universe of discourse and represent the set of all users who have rated item $i$. Then, the fuzzy set $A$ in $X$ is defined as $A=\left\{\left(x, \mu_{A}(x)\right): x \in X\right\}$.

For $x \in X$, the membership grade $\mu_{A}(x)$ is judged by the defined conditions as follows:
$\mu_{A}(x)= \begin{cases}1, & \text { if } r_{x i}>\overline{r_{x}} \\ 0, & \text { if } r_{x i} \leq \overline{r_{x}}\end{cases}$

where $r_{x i}$ is the rating that user $x$ rates on item $i$, and $\overline{r_{x}}$ is the average value rated by user $x$. According to the relationship between $r_{x i}$ and $\bar{r}_{x}$, user preferences are divided into two categories. Specifically, when $r_{x i}>\overline{r_{x}}$, it means user $x$ likes item $i$ to a certain extent. While, it implies that user $x$ dislikes item $i$.

However, in the real recommendation systems, each user will be affected by the corresponding surrounding environment. Thus, users who rate the same item affect each other. In other words, although supporters and opponents have different preferences (like or dislike) on the same item, they always influence each other in the process of propagation, so that a certain degree of hesitation will be produced in user preferences. The specific process is as follows. On the one hand, the preference of supporters will affect opponents. We can represent this as $\mu_{A}(x) \cdot\left[1-\mu_{A}(x)\right]$. On the other hand, the preference of opponents will also affect supporters, and then we mark it as $\left[1-\mu_{A}(x)\right] \cdot \mu_{A}(x)$. Therefore, the relationship of their mutual influence makes these users who will rate the same item be affected when making decisions, showing a certain degree of hesitation in the real recommendation systems. That is constructed by the sum of the above two values as $2 \cdot \mu_{A}(x) \cdot\left[1-\mu_{A}(x)\right]$.

To stress the effect of hesitation in decision-making, the defined fuzzy set $A$ is extended to a vague set according to the concept of influence propagation [12] among users described above. As an extension and improvement theory of fuzzy sets, vague sets [34] have the merit that it sufficiently includes actions of like, dislike, and hesitation.

Definition 2 (Vague sets) The vague set $\tilde{A}$ in $X$ is given by $\tilde{A}=\left\{\left(x, t_{\tilde{A}}(x), 1-f_{\tilde{A}}(x): x \in X\right)\right\}$, with the condition $t_{\tilde{A}}(x)+f_{\tilde{A}}(x)+\pi_{\tilde{A}}(x)=1$. Where $t_{\tilde{A}}(x)$ is described as the degree of membership, $f_{\tilde{A}}(x)$ is termed as the degree of nonmembership, and $\pi_{\tilde{A}}(x)$ represents the degree of hesitation.

According to the degree of hesitation caused by influence propagation analyzed above and the condition $t_{\tilde{A}}(x)+f_{\tilde{A}}(x)+\pi_{\tilde{A}}(x)=1$ on vague set $\tilde{A}$, we can infer that the degree of membership $t_{\tilde{A}}(x)$ is obtained by the degree of membership $\mu_{A}(x)$ on the defined fuzzy set to remove the preference influence of opponents on supporters. The formula is expressed as $t_{\tilde{A}}(x)=\mu_{A}(x)-\left[1-\mu_{A}(x)\right] \cdot \mu_{A}(x)=\mu_{A}^{2}(x)$. Similarly, we can deduce that the degree of non-membership $f_{\tilde{A}}(x)$ is gained by removing the preference influence of supporters on opponents from the degree of membership $\mu_{A}(x)$ on our defined fuzzy set. That is $f_{\tilde{A}}(x)=\left[1-\mu_{A}(x)\right]-\mu_{A}(x) \cdot\left[1-\mu_{A}(x)\right]=\left[1-\mu_{A}(x)\right]^{2}$. In addition, it should be noted from the attribute of vague sets that the interval centre contains important information. The expression of interval centre is $\varphi_{\tilde{A}}(x)=\frac{\mu_{\tilde{A}}(x)-\left[1-f_{\tilde{A}}(x)\right]}{2}$. 


\subsubsection{KL Divergence Between Vague Sets}

After completing aforementioned definitions of fuzzy sets and vague sets on recommendation system. We first compute user preference probability on fuzzy set and vague set, respectively. And then, KL divergence is introduced to estimate the difference between vague sets.

According to the membership grade $\mu_{A}(x)$ of each user on fuzzy set $A$, we first calculate $p_{A, l}$ and $p_{A, d}$, which indicate the probability that these users have optimistic attitude on item $i$ and the probability that these users have pessimistic attitude on item $i$, respectively:

$\left\{\begin{array}{l}p_{A, l}=\frac{\sum_{x \in X} \mu_{A}(x)}{N(X)} \\ p_{A, d}=1-p_{A, l}\end{array}\right.$

where $N(X)$ represents the number of users in the universe $X$.

Based on user preference probability on fuzzy set $A$, we obtain $p_{\tilde{A}, l}, p_{\tilde{A}, d}, p_{\tilde{A}, h}$ and $p_{\tilde{A}, m}$, which mean the probability that these users support item $i$, the probability that these users opposite item $i$, the probability of hesitation, and the probability of interval centre on vague set $\tilde{A}$ :

$\left\{\begin{array}{l}p_{\tilde{A}, l^{\prime}}=p_{A, l}-p_{A, l} \cdot p_{A, d} \\ p_{\tilde{A}, d^{\prime}}=p_{A, d}-p_{A, l} \cdot p_{A, d} \\ p_{\tilde{A}, h}=2 \cdot p_{A, l} \cdot p_{A, d} \\ p_{\tilde{A}, m}=\frac{p_{\tilde{A}, l^{\prime}}+\left[1-p_{\tilde{A}, d^{\prime}}\right]}{2}\end{array}\right.$

We aim to construct a well-suited similarity measure from the perspective of user preference probability to calculate the similarity value between vague sets, that is, the similarity value between corresponding items. It is well-known that divergence is a very important concept of probability statistics. The divergence theory $[10,22]$ is a powerful tool that has been recognized to measure differences between information. Therefore, the KL divergence is utilized to evaluate the distance between any two vague sets $(\tilde{A}$ and $\tilde{B})$. The KL distance can be expressed in Eq. (12), which breaks the constraint of co-rated cases, and can calculate the similarity between any two items by making full use of the rating information. Therefore, it has good adaptability to sparse data:

$\mathrm{KL}(\tilde{A} \| \tilde{B})=\sum_{\mathrm{pre}^{\prime} \in\left\{l^{\prime}, d^{\prime}, h, m\right\}} p_{\tilde{A}, \mathrm{pre}^{\prime}} \cdot \log _{2} \frac{p_{\tilde{A}, \mathrm{pre}^{\prime}}}{p_{\tilde{B}, p r e^{\prime}}}$

where the set of user preferences and interval centre on vague set is $\left\{l^{\prime}, d^{\prime}, h, m\right\}$, and pre' represents any element in this set.

However, it is worth noting that when $p_{\tilde{A} \text {,pre' }}=0$ or $p_{\tilde{B}, \text { pre }}=0$, the formula 12 will make no sense. To remedy it, we smooth the user preference probability $p_{\text {pre' }}$ to obtain a new user preference probability $\hat{p}_{\text {pre' }}$, as shown in the following Eq. (13):

$\hat{p}_{\mathrm{pre}^{\prime}}=\frac{\delta+p_{\mathrm{pre}^{\prime}}}{1+\delta \cdot|K|}$

where the smoothing factor is $\delta$ with $0<\delta<1 .|K|$ represents length of the set to which pre' belongs. We derive the error between $p_{\mathrm{pre}^{\prime}}$ and $\hat{p}_{\mathrm{pre}^{\prime}}$ after smoothing, as shown below.

$$
\begin{aligned}
\text { error } & =\left|\hat{p}_{\mathrm{pre}^{\prime}}-p_{\mathrm{pre}^{\prime}}\right| \\
& =\left|\frac{\delta+p_{\mathrm{pre}^{\prime}}}{1+\delta \cdot|K|}-p_{\mathrm{pre}^{\prime}}\right| \\
& =\left|\frac{\delta-p_{\mathrm{pre}^{\prime}} \cdot \delta \cdot|K|}{1+\delta \cdot|K|}\right| \\
& =\left|\frac{1-p_{\mathrm{pre}^{\prime}} \cdot|K|}{1 / \delta+|K|}\right|
\end{aligned}
$$

According to this above deduction of equation, if $\delta \rightarrow 0$, then error $\rightarrow 0$. Thus, $\hat{p}_{\text {pre' }}$ is a reasonable substitute for $p_{\mathrm{pre}^{\prime}}$ when $\delta$ is set to a small value. Here, we assumed that $\delta=0.000009$.

Finally, KL distance after the process of smoothing can be rewritten as follows:

$\operatorname{KL}(\tilde{A} \| \tilde{B})=\sum_{\operatorname{pre}^{\prime} \in\left\{l^{\prime}, d^{\prime}, h, m\right\}} \hat{p}_{\tilde{A}, \mathrm{pre}^{\prime}} \cdot \log _{2} \frac{\hat{p}_{\tilde{A}, \mathrm{pre}^{\prime}}}{\hat{p}_{\tilde{B}, \mathrm{pre}^{\prime}}}$

\subsubsection{The impact of rating quantity}

In the above-mentioned KL divergence based on vague sets, only probability values after fraction reduction are involved in the calculation of KL divergence, resulting in a certain loss of the information of rating quantity. Especially in the case of extremely sparse data, it is easy to cause large errors in similarity results. In general, the more users who have rated a given item, the more correct the probability information we can get. To make similarity results more credible, the effect of the number of ratings needs to be considered when calculating KL divergence. Thus, we define a weight factor $\alpha$ to represent the ratio of vague set $\tilde{A}$, using the ratio of the scale of universe on vague set $\tilde{A}$ to the sum of the scales of universe on vague sets $\tilde{A}$ and $\tilde{B}$. Correspondingly, the ratio of vague set $\tilde{B}$ is $1-\alpha$ :

$\alpha=\frac{N(\tilde{A})}{N(\tilde{A})+N(\tilde{B})}$ 
where $\alpha$ is dynamic as the scale of universe on the vague set varies. It efficiently reflects the difference about the number of ratings between vague sets.

Therefore, the distance formula after joining the number of ratings is expressed in Eq. (17), which avoids a certain loss of the information of rating quantity caused by fraction reduction in the calculation of KL divergence and improves the accuracy of similarity values in sparse data:

$$
\mathrm{KL}(\tilde{A} \| \tilde{B})=\sum_{\operatorname{pre}^{\prime} \in\left\{l^{\prime}, d^{\prime}, h, m\right\}} \alpha \cdot \hat{p}_{\tilde{A}, \mathrm{pre}^{\prime}} \cdot \log _{2} \frac{\alpha \cdot \hat{p}_{\tilde{A}, \mathrm{pre}^{\prime}}}{(1-\alpha) \cdot \hat{p}_{\tilde{B}, \mathrm{pre}^{\prime}}}
$$

On the basis of the dual concept of the distance and similarity measure, we measure the similarity of items $i$ and $j$ by calculating KL distance between corresponding vague sets $\tilde{A}$ and $\tilde{B}$. The formula is indicated as below:

$\operatorname{sim}(i, j)=\frac{1}{1+\operatorname{KL}(\tilde{A} \| \tilde{B})}$ neighbors set $(N)$ of the target item $i$ into two subsets ( $N 1$ and $N 2$ ) by judging whether the given user $u$ rates them. First, items are classified as subset $N 1$ by satisfying this condition. Any rating $r_{u j}$ exists and can directly participate in the prediction process. Second, items that do not meet this condition are categorized as subset $N 2$. User $u$ has not already rated any item in subset $N 2$ and cannot be directly involved in predictive calculation. Aiming at this case, the approximate substitution principle is further introduced to indirectly allow these neighbors to participate in prediction process. For any item $j$ from subset $N 2$, we search user $v$ in the set of users who have rated item $j$, and ensure $v$ is the most similar to the given user $u$. Then, the non-given rating $r_{u j}$ is replaced with the estimated rating $r_{v j}$. However, $r_{v j}$ is not as accurate as the explicitly given value $r_{u j}$. Hence, $\operatorname{sim}(u, v)$ is further added as the specified weight factor to adjust the error caused by this approximate substitution principle.

Formally, our proposed prediction method is defined in Eq. (19), which improves the ability of prediction in sparse data by integrating more rating information of neighbors:

$p_{u i}=\bar{r}_{i}+\frac{\sum_{j \in N 1} \operatorname{sim}(i, j) \cdot\left(r_{u j}-\bar{r}_{j}\right)+\sum_{j \in N 2, v \in N(u)} \operatorname{sim}(u, v) \cdot \operatorname{sim}(i, j) \cdot\left(r_{v j}-\bar{r}_{j}\right)}{\sum_{j \in N 1} \operatorname{sim}(i, j)+\sum_{j \in N 2, v \in N(u)} \operatorname{sim}(u, v) \cdot \operatorname{sim}(i, j)}$

\subsection{Item-Based Prediction Method with the New Neighbor Selection Strategy}

In the traditional item-based prediction methods, the availability of neighbors is strictly required in prediction formulas. To generate prediction, $r_{u j}$ needs to be explicitly given, that is, a given user must rate the nearest neighbors (items). Due to sparse data, a considerable number of neighbors do not meet the constraint condition of prediction formulas. Even though these neighbors are very similar to the target item, their information is not effectively applied for prediction, either. This phenomenon will decrease the accuracy of prediction.

Based on aforementioned analysis, our reasonable assumption is that these neighbors may still make a valuable contribution to the prediction process, although the given user does not explicitly rate them. This is a promising process ignored in traditional prediction methods. However, it should be considered in our algorithm to effectively deal with the problem of sparse data. Inspired by this assumption, we propose an item-based prediction method with the new neighbor selection strategy. The detailed implementation process is as follows. We divide these items in the nearest where $N(u)$ represents the nearest neighbors of user $u$, and user $v$ is the most similar to user $u$ in $N(u) \operatorname{sim}(u, v)$ is the same as the calculation principle of $\operatorname{sim}(i, j)$ in our proposed similarity measure, and the process of detailed implementation can be omitted.

It is necessary to point out two extreme cases in our proposed prediction method. If a given user has rated each item in the nearest neighbors of item $i$, the prediction formula is equivalent to the traditional prediction formula MC. On the contrary, if a given user has not rated any of nearest neighbors of the target item, our proposed prediction formula evolves into the following:

$p_{u i}=\bar{r}_{i}+\frac{\sum_{j \in N(i), v \in N(u)} \operatorname{sim}(u, v) \cdot \operatorname{sim}(i, j) \cdot\left(r_{v j}-\bar{r}_{j}\right)}{\sum_{j \in N(i), v \in N(u)} \operatorname{sim}(u, v) \cdot \operatorname{sim}(i, j)}$

Finally, the item-based CF model (ISP) in this paper is composed of the item similarity measure based on vague sets and prediction method with the new neighbor selection strategy. The detailed implementation process is presented in Algorithm 1. 


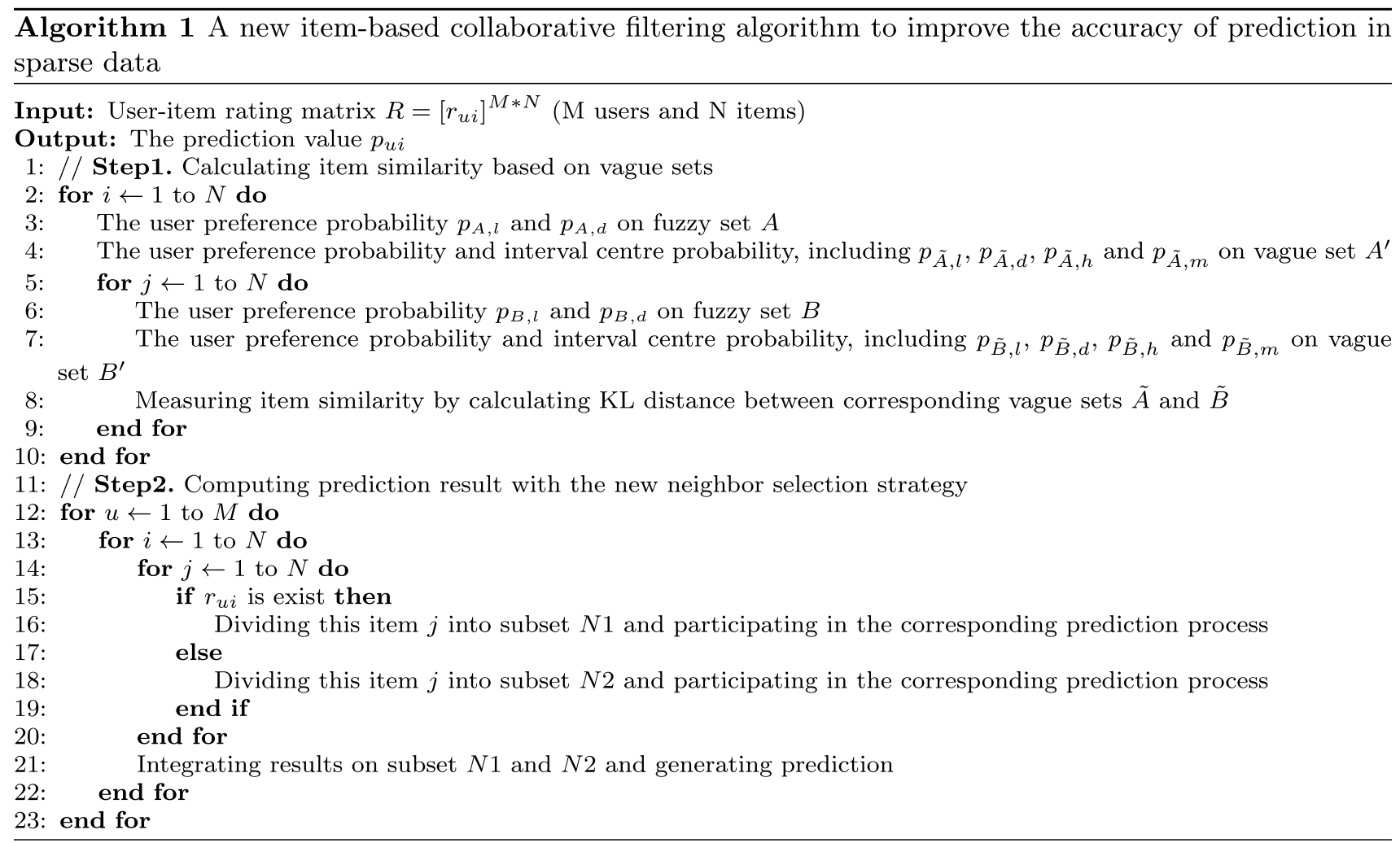

\section{Experiment}

\subsection{Experimental Setup}

In this section, we carry out all experiments on the development platform AI Studio, where it has a quad-core processor, primary memory is $32 \mathrm{~GB}$, the development language is Python3.7, and the framework version is PaddlePaddle 2.0.2.

\subsection{Data Preparation}

For our experiments, two commonly-used benchmark data sets, namely MovieLens100K (ML-100K) and MovieLens1M (ML-1M) both from MovieLens, are employed. The properties, including number of users, number of items, number of ratings and sparsity, are briefly describes in Table 1. The experimental results of each algorithm are

Table 1 Data sets properties

\begin{tabular}{lllll}
\hline Data set & Users & Items & Ratings & Sparsity \\
\hline ML-100K & 943 & 1682 & 100000 & $93.7 \%$ \\
ML-1M & 6040 & 3952 & 1000209 & $95.8 \%$ \\
\hline
\end{tabular}

verified according to the fivefold cross-validation method. Specifically, we classify each data set into two parts: $80 \%$ of rating data is included in the training set, and the rest is regarded as the testing set.

\subsection{Evaluation Indicators}

We evaluate the prediction accuracy [11, 29] based on Mean Absolute Error (MAE) and Root Mean Square Error (RMSE). The two metrics compute the error by comparing the difference of predicted values and actual values. And lower errors mean the better prediction accuracy. The formulas of MAE and RMSE are shown in Eqs. (21) and (22), respectively:

$\mathrm{MAE}=\frac{1}{n} \sum_{i=1}^{n}\left|r_{u i}-p_{u i}\right|$

$\mathrm{RMSE}=\sqrt{\frac{1}{n} \sum_{i=1}^{n}\left(r_{u i}-p_{u i}\right)^{2}}$

where $r_{u i}$ is the actual rating in the testing set. $n$ represents the number of prediction results. 
We assess the recommendation accuracy $[3,8]$ using F1-value. As a comprehensive indicator, it integrates Precision and Recall simultaneously. The higher F1-value indicates the better recommendation quality. The expressions of Precision, Recall and F1-value are as follows, respectively:

Precision $=\frac{n\left(I_{p r} \cap I_{a r}\right)}{n\left(I_{p r}\right)}$

Recall $=\frac{n\left(I_{p r} \cap I_{a r}\right)}{n\left(I_{a r}\right)}$

$\mathrm{F} 1-$ value $=\frac{2 \times \text { Precision } \times \text { Recall }}{\text { Precision }+ \text { Recall }}$

where $I_{a r}$ and $I_{p r}$ are the real recommendation list and the predicted recommendation list, respectively. $n(\cdot)$ represents the length of specified list. The recommendation rule adopted is that each rating needs to be larger than the average value rated by corresponding user.

In addition to the above two types of evaluation indicators, we also present the rate of successful predictions (RSP) and perfect predictions (RPP) [37] to comprehensively measure the prediction ability of algorithms. RSP emphasizes the ability of a $\mathrm{CF}$ algorithm to make valid predictions, regardless of whether these results are accurate or not. RPP refers to the rate of correctly predicting the actual ratings in the testing set, which stresses the ability to make correct predictions. Their formulas are defined as below:

$\mathrm{RSP}=\frac{N_{s}}{N_{t}}$

$\mathrm{RPP}=\frac{N_{p}}{N_{t}}$

where $N_{s}$ and $N_{p}$ represents the number of successful predictions and correct predictions, respectively. In addition, $N_{t}$ is the amount of ratings that needs to be predicted.

\subsection{Experimental Results and Analysis}

In this section, related experiments are mainly divided into two parts to validate the performance of our proposed model ISP. The first part is to gradually test the effectiveness of similarity measure and prediction method designed in ISP algorithm. The second part is to compare and discuss the performance of ISP and other state-of-the-art algorithms in each indicator. All experiments are carried out in ML-100K and ML-1M data sets, respectively. The number of the nearest neighbors, K, ranges from 10 to 100 in steps of 10 .

\subsubsection{The Effectiveness Test of the Proposed Model}

According to the steps of the proposed model ISP, we first construct different algorithms, namely, case1, case2, and case3, respectively. Then, the effectiveness of each component in ISP algorithm is gradually analyzed in experiments. The details of these three cases are as follows.

Case 1: according to the definition of vague set, we first calculate and smooth user preference probability, and then these probability values are added to the KL divergence to measure the difference between vague sets, as shown in formula (15). Based on the formula (18), we obtain the corresponding item similarity. The widely used MC is selected as the prediction method.

Case 2: based on the item similarity in case 1, the weight factor $\alpha$ is defined to further consider the impact of rating quantity, which is incorporated in KL divergence to obtain a new distance formula as shown in (17). Then, we obtain the corresponding item similarity through formula (18). MC is still selected as the prediction method.

Case 3: the similarity measure in case 2 is still adopted to compute item similarity. The prediction method MC used above is replaced by the proposed prediction formula (19).

Here, MC is selected as the comparative method of our proposed prediction method. The main reason is MC performs more efficient than other traditional prediction methods, which has proved by a comparative study in [27]. And it should be noted that case 3 algorithm is our proposed model ISP.

First, case 1 , case 2 and case 3 algorithms are all carried out in ML-100K data set. The results of prediction accuracy are given in Fig. 1a, b, respectively. Prediction errors (MAE and RMSE) of all algorithms decline gradually with the increasing of $K$ values. Compared with case1, case 2 algorithm has an approximate reduction of $1-5 \%$ in MAE and 3-8\% in RMSE. The main reason is that the rating quantity is considered to avoid the information loss caused by fraction reduction in case 2 algorithm, which conduces to enhancing the prediction performance. Compared with case2, the MAE and RMSE of case3 algorithm decrease nearly $2 \%$ in different $K$ values. When $\mathrm{K}$ value exceeds 40 , prediction errors of case 3 gradually become steady. At this time, MAE is about 0.745 and RMSE is about 0.958. Especially when the number of neighbors is relatively small, the advantage of prediction results is more prominent. The primary reason is that the traditional prediction method MC in case 2 algorithm is replaced by our proposed prediction method in case 3 algorithm. When generating prediction, the rating information of the nearest neighbors can participate to adjust the final prediction result, which further improves the accuracy of prediction.

Figure 1c shows the results of recommendation accuracy about case 1 , case 2 and case 3 algorithms. With the increasing 


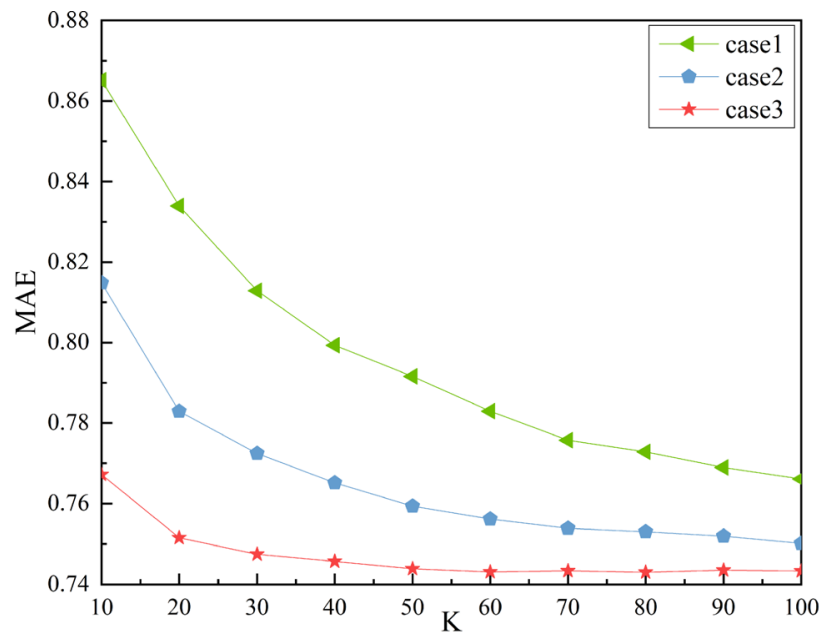

(a) MAE at different $K$ values.

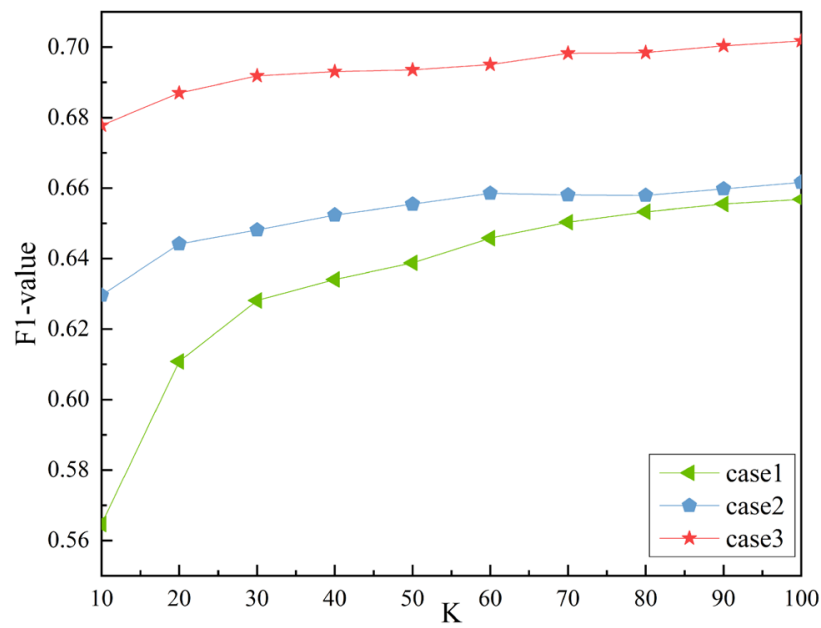

(c) F1-value at different $K$ values.

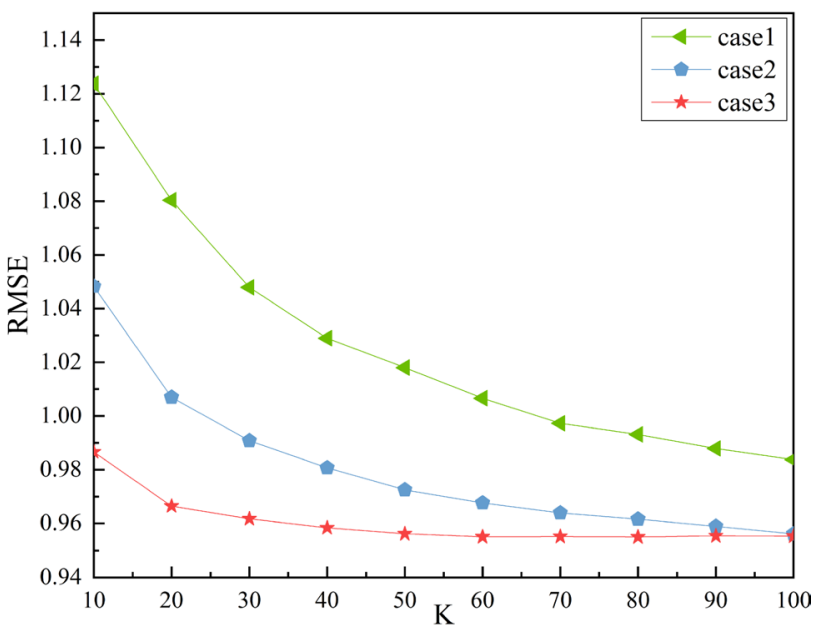

(b) RMSE at different $K$ values.

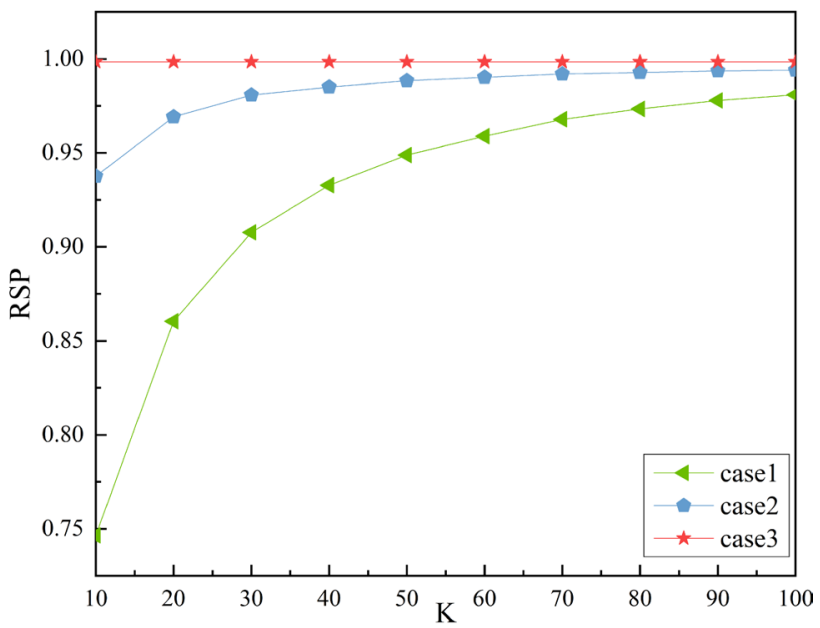

(d) RSP at different $K$ values.

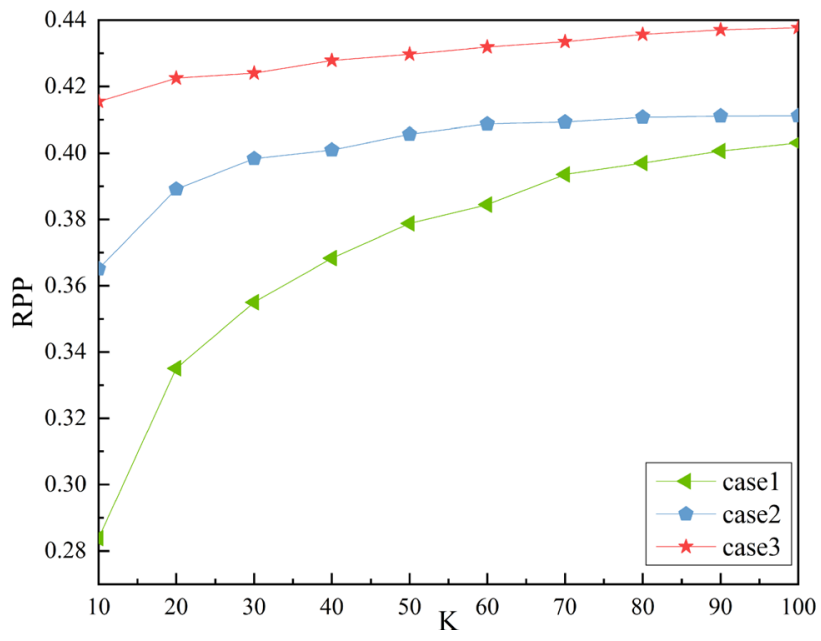

(e) RPP at different $\mathrm{K}$ values

Fig. 1 Performance results in ML-100K 
of the number of neighbors, F1-value of each algorithm increases continuously. It is worth pointing out that the interval range of case1 has an obvious change, while case 2 and case 3 are relatively stable as the number of neighbors varies. Compared with case1 algorithm, F1-value of case2 has increased by $1.87 \%$ on average. Likewise, F1-value of case 3 has increased by an average of $4.11 \%$ compared with case 2 algorithm. Therefore, we can infer that case 3 algorithm has the best recommendation accuracy in different $\mathrm{K}$ values, which proves the effectiveness of the number of ratings considered in the proposed similarity measure and prediction method.

The prediction ability of case 1 , case 2 and case 3 algorithms are depicted in Fig. 1d, e, respectively. As we can see from Fig. 1d, in different number of the nearest neighbors, the RSP value of case 2 is evidently higher about $5.71 \%$ on average than case 1 algorithm. Meanwhile, the RSP value of case 3 has a slight rise about $1.61 \%$ compared with case 2 algorithm. What's more, with the increasing of neighbors, the gap between case 2 and case 3 becomes smaller. Case 3 algorithm has the highest RSP value about 0.998 and remains unchanged. Figure 1e illustrates that case 2 obtains the better RPP value compared with case 1 algorithm, and the RPP value of case 3 has better performance compared with case 2 algorithm. The main reason is that the similarity measure is not limited by co-rated cases, and can calculate similarity results between any items. Meanwhile, our proposed prediction method does not strictly demand that a given user rates the nearest neighbors of the target item, either. Therefore, there are few restrictions in calculating predictive values.

In the same way, these three algorithms are executed in ML-1M data set, Figure 2 depicts the results of each index. The similar results are obtained that the performance of case 2 algorithm obviously outperforms case 1 , and the performance of case 3 algorithm is better than case2. Therefore, according to the analysis of in the above two data sets with different degree of sparsity, case 3 algorithm has the best performance, which also proves the effectiveness of each component in our proposed model.

\subsubsection{Comparative Analysis}

In this section, to further validate the performance of our proposed model ISP, other state-of-the-art CF algorithms, including SPCC [16], JMSD [4], NHSM [15], BCF [21], KLCF [28], $\alpha$-CF [30], RJMSD [3] and OS [13], are implemented in MovieLens data sets. We mainly discuss the results of ISP and these comparison algorithms in different number of the nearest neighbors.

MAE and RMSE Figure 3 portrays the results of prediction accuracy in ML-100K data set. As the increasing of $\mathrm{K}$ values, prediction errors of all algorithms decrease gradually. ISP algorithm remains the lowest MAE and RMSE values in different $K$ values. When the number of neighbors is less than 50, our proposed model has an average reduction of $2.6 \%$ in MAE and 3.2\% in RMSE compared with the closest competitor (NHSM). The principal reason is that ISP algorithm integrate more ratings when calculating item similarity, to make the nearest neighbors more reliable. The prediction stage can also sufficiently apply the ratings information of the nearest neighbors to further improve the accuracy of the predictive values. Likewise, the results of prediction accuracy in ML-1M data set are portrayed in Fig. 4. In different number of neighbors, ISP algorithm attains the lowest prediction errors compared with other algorithms. When the number of neighbors is 100 , the values of MAE and RMSE achieve optimal, which are about 0.712 and 0.916 , respectively.

\section{F1-value}

Figures 5 and 6 illustrate the results of recommendation accuracy in ML-100K and ML-1 M data sets, respectively. As shown in Fig. 5, with the number of neighbors increases, F1-value of each algorithm becomes continuously larger. In different number of neighbors, ISP algorithm always remains the best F1-value. When $K$ value is 100 , the corresponding best F1-value is close to 0.702 . It is worth noting that, compared with other comparison algorithms, the F1-value of BCF and KLCF algorithms has significantly improved. Furthermore, there is only a gap about $1 \%$ between these two algorithms and ISP. According to the above analysis, we can conclude that the similarity measure based on probability distribution is an outstanding way to enhance the accuracy of recommendation. Similarly, Fig. 6 shows that ISP attains the best F1-value than other comparison algorithms in different number of the nearest neighbors. However, the F1-value of NHSM and JMSD algorithms performs well and keeps the closest trend to ISP algorithm. The possible reason is that the quantity information of co-rated cases has an important impact on the recommendation results in extremely sparse data sets. NHSM and JMSD algorithms emphasize the proportion of co-rated cases, so they have better recommendation quality than other comparison algorithms.

RSP and RPP The RSP and RPP results in ML-100K and ML-1M data sets are described in Figs. 7 and 8, respectively. As illustrated in Fig. 7a, the RSP values of ISP algorithm achieve the best and remain about 0.998 in different $\mathrm{K}$ values. Among all these comparison algorithms, BCF and KLCF algorithms have better RSP values and behave basically consistent. An important reason is that these two approaches can calculate similarity results between any users. Therefore, computation results are more objective and contribute to generating more predictions. From Fig. 7b, it can be seen that the RPP values of all algorithms become higher with the increasing of K values. The RPP value of ISP algorithm has optimal performance with the range from 


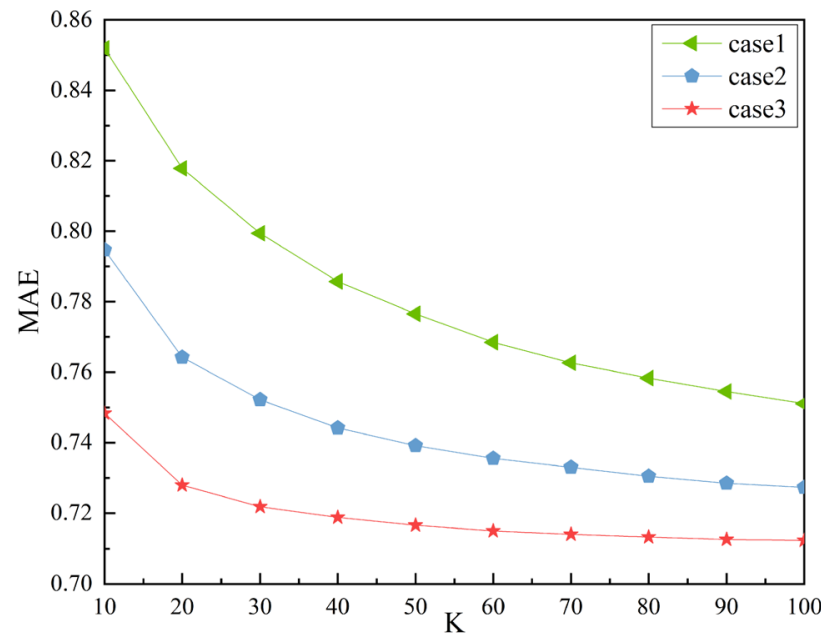

(a) MAE at different $K$ values.

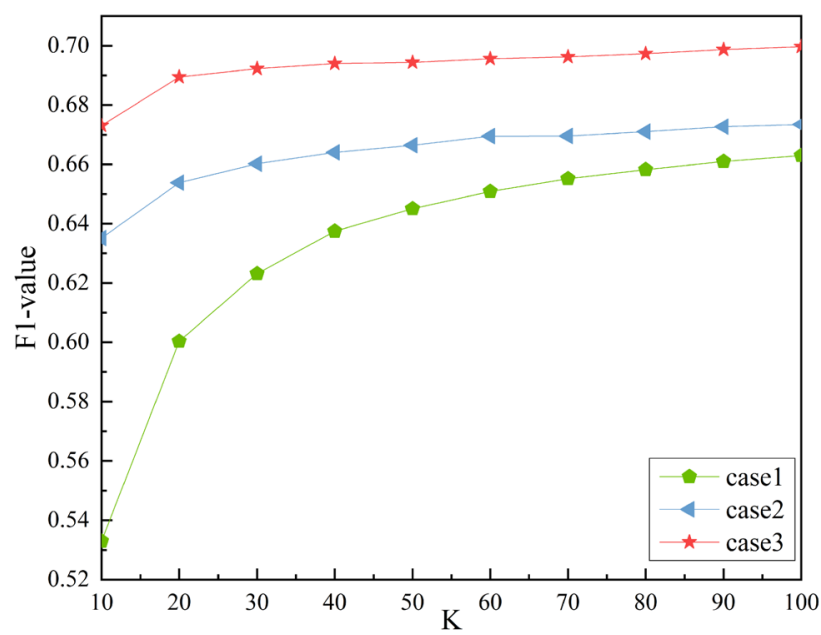

(c) F1-value at different $K$ values.

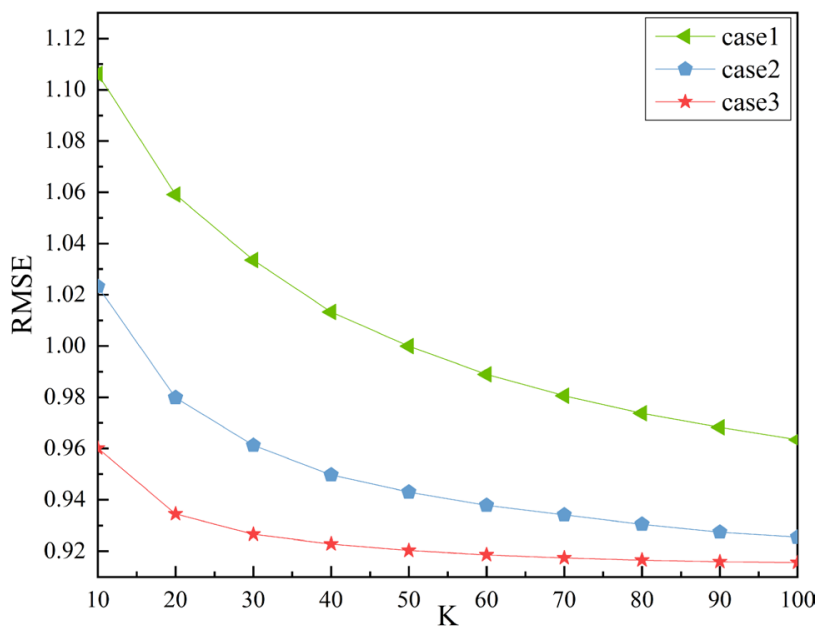

(b) RMSE at different $K$ values.

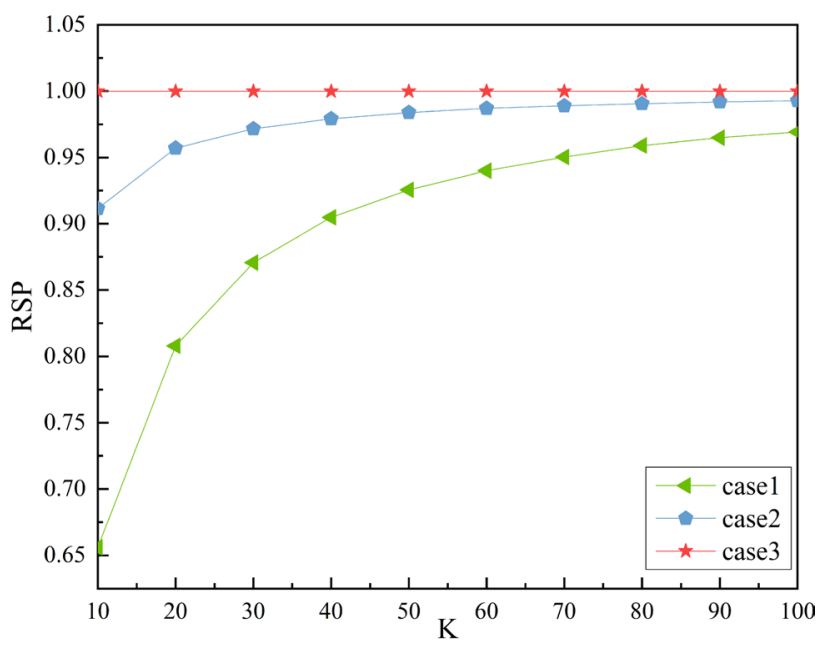

(d) RSP at different $K$ values.

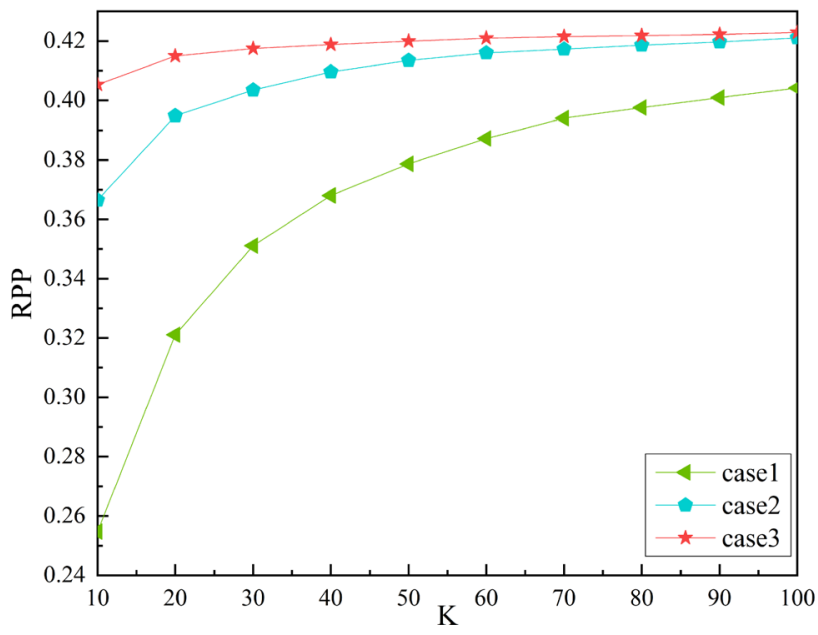

(e) RPP at different $\mathrm{K}$ values.

Fig. 2 Performance results in ML-1 M 


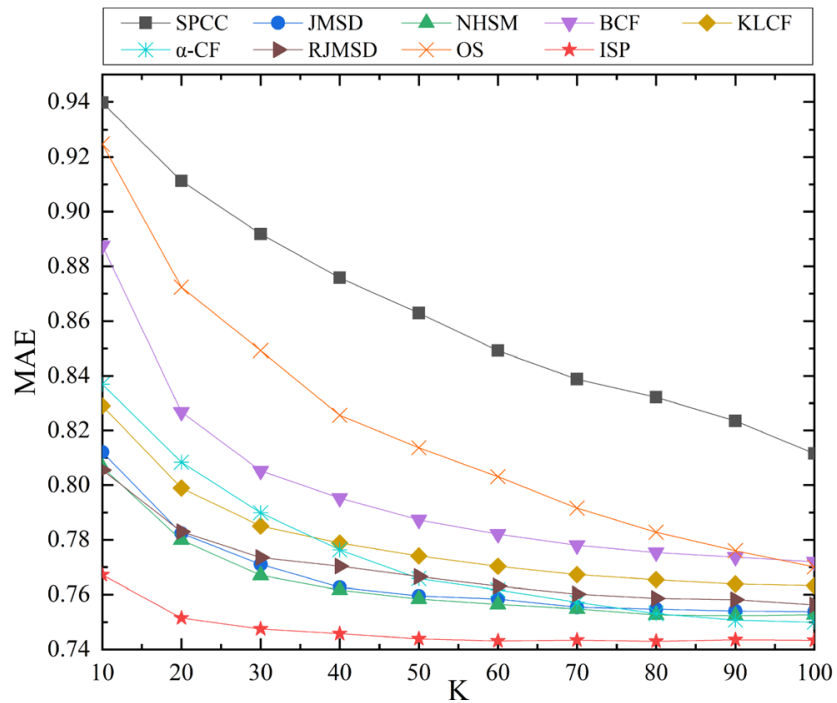

(a) MAE of all algorithms in ML-100K.

Fig. 3 MAE and RMSE values of all algorithms in ML-100K

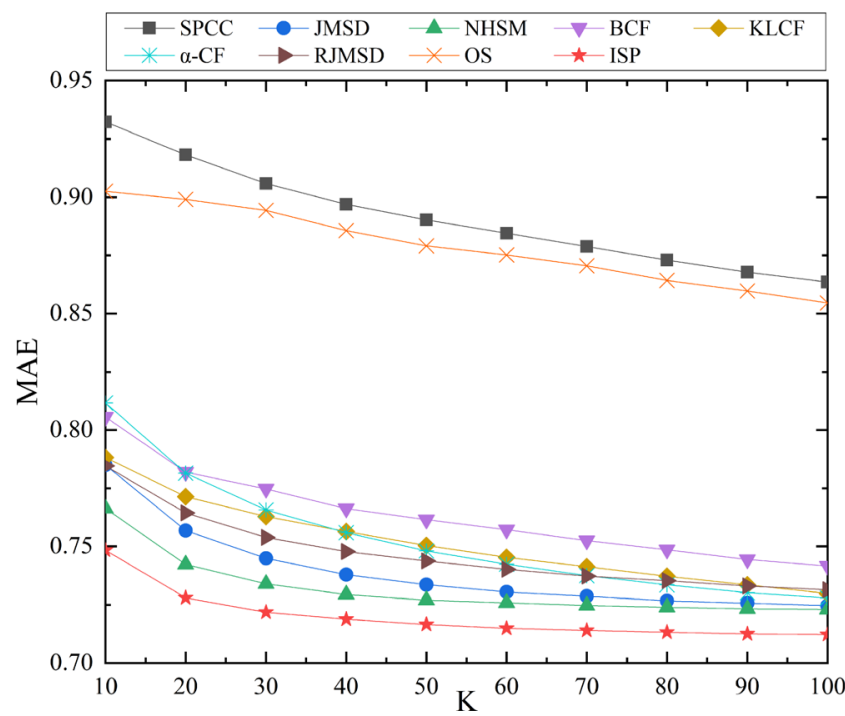

(a) MAE of all algorithms in ML-1M.

Fig. 4 MAE and RMSE values of all algorithms in ML-1 M

0.41 to 0.44 . Meanwhile, the RPP values of NHSM and KLCF algorithms are closest to ISP, and the distribution intervals both are located between 0.37 and 0.42 . Similarly, as can be seen from Fig. 8a, the RSP value of ISP is an invariant constant about 0.999. Meanwhile, the RSP values of NHSM, BCF and KLCF algorithms are gradually larger with the increasing of $\mathrm{K}$ values, and the gap with ISP algorithm becomes unapparent. Especially when $\mathrm{K}$ value is 100 , the gap between them is less than $1 \%$. From Fig. 8 b, we can conclude that, with the number of neighbors increases,

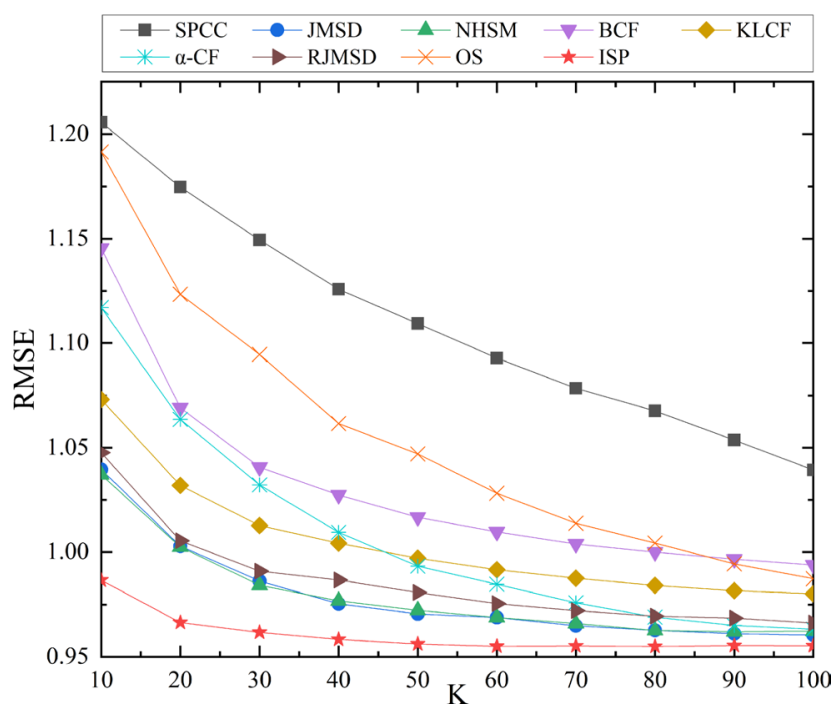

(b) RMSE of all algorithms in ML-100K.

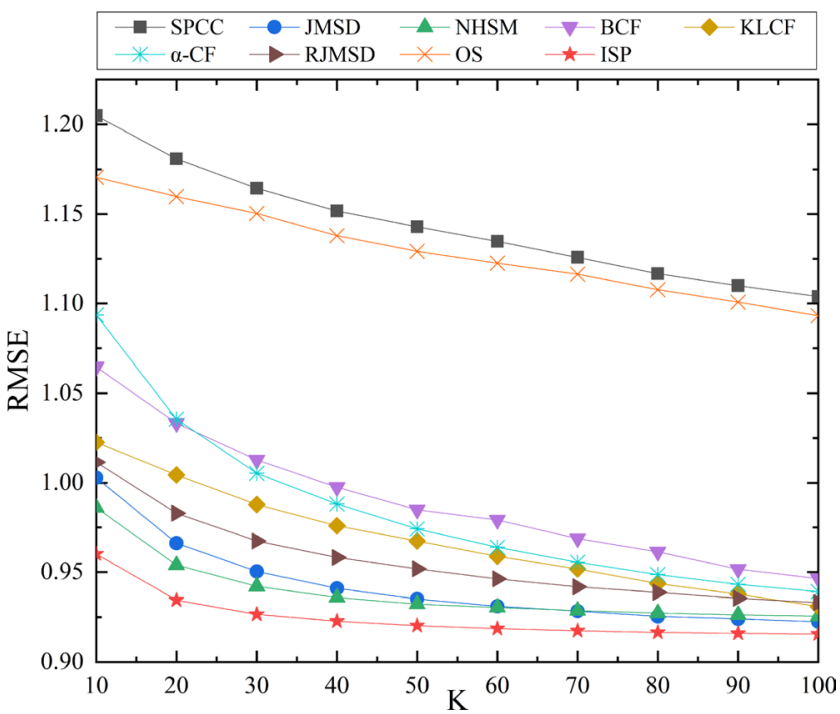

(b) RMSE of all algorithms in ML-1M.

ISP algorithm always maintains the highest RPP value. In particular, when $K$ value is less than 50, ISP is apparently superior to other comparison algorithms.

\section{Conclusion}

In this paper, to enhance the adaptability to sparse data, we propose an item-based CF model (ISP) composed of a new item similarity measure and prediction method. First, the 


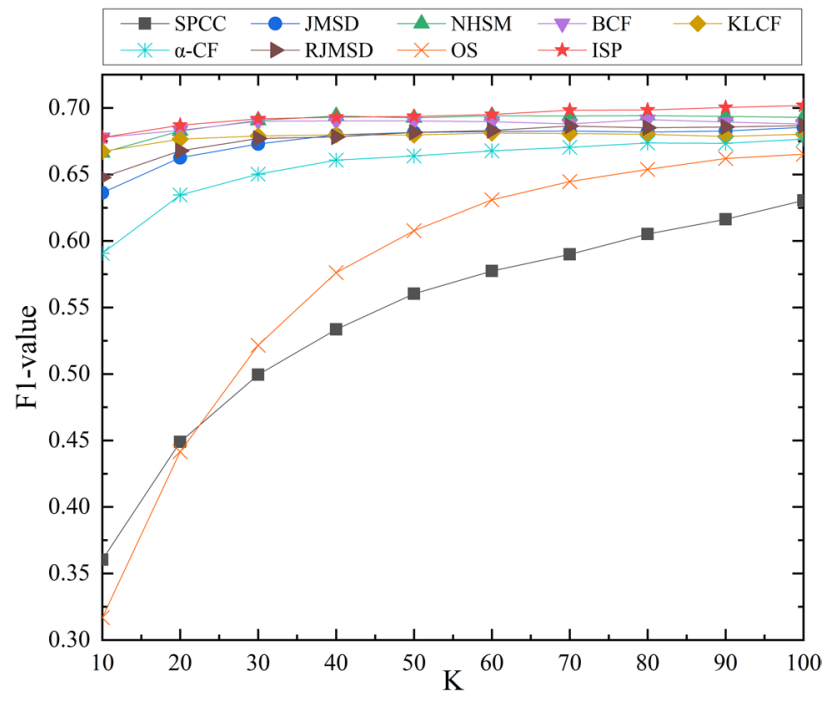

Fig. 5 F1-value of all algorithms in ML-100K

association between fuzzy sets and recommendation systems is introduced. Based on this association, fuzzy sets on recommendation systems are defined and extended to vague sets. Next, we use KL divergence to calculate the similarity between vague sets from the perspective of user preference probability, that is, the similarity between corresponding items. To further improve the accuracy of similarity results, the impact of rating quantity is considered in the similarity measure. What's more, an item-based prediction method with the new neighbor selection strategy is proposed by

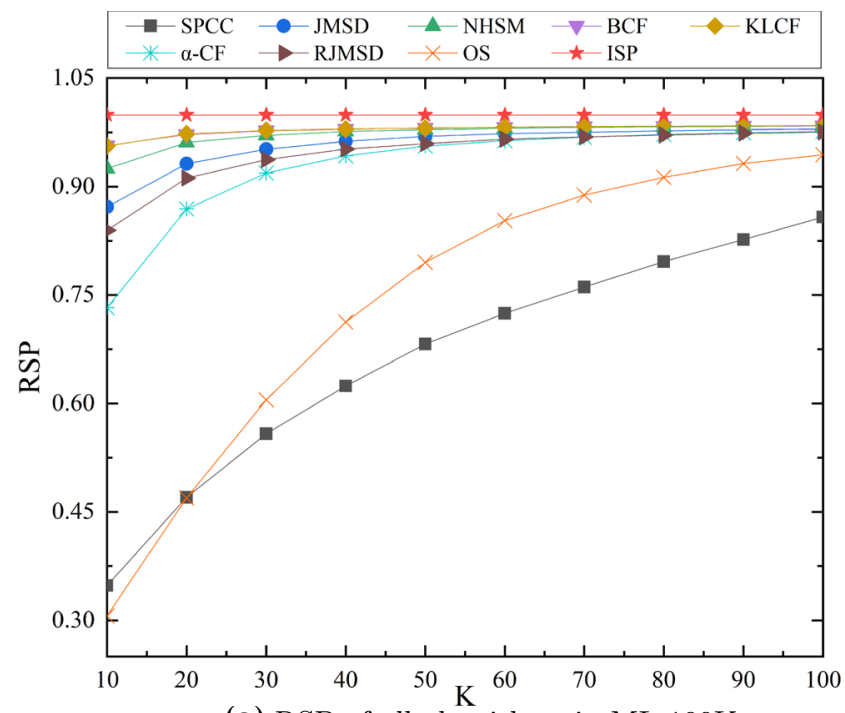

(a) RSP of all algorithms in ML-100K.

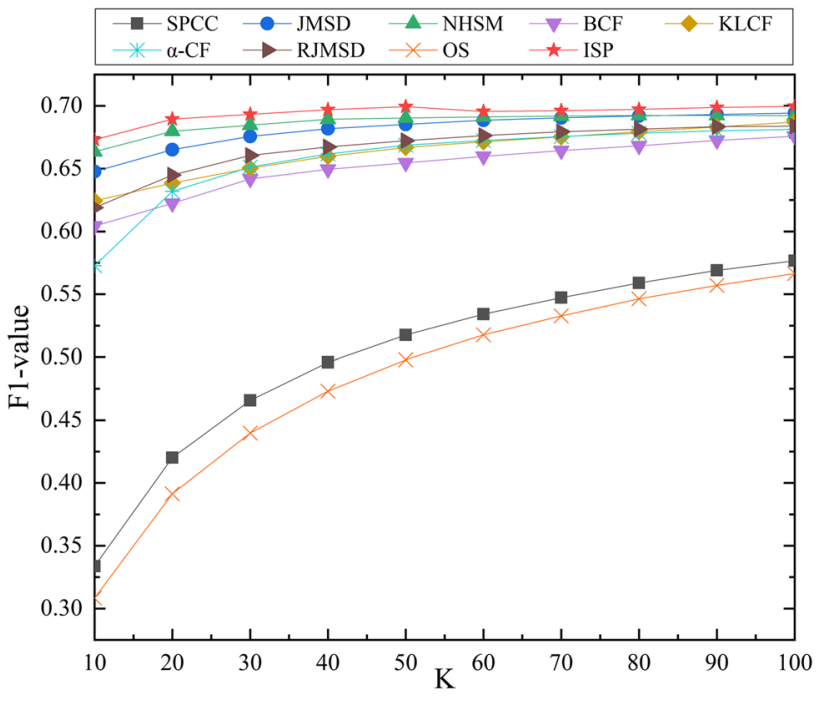

Fig. 6 F1-value of all algorithms in ML-1 M

aggregating more potentially valuable nearest neighbors' information to achieve higher prediction accuracy. Experiments on two data sets with different sparsity show that our ISP algorithm is less affected by data sparsity and can provide better recommendation performance.

For future work, we intend to explore how to better deal with the problem of data sparsity. Meanwhile, a relationship between accuracy and efficiency will be investigated to obtain better prediction and recommendation quality while maintaining less time cost.

Fig. 7 RSP and RPP values of all algorithms in ML-100K

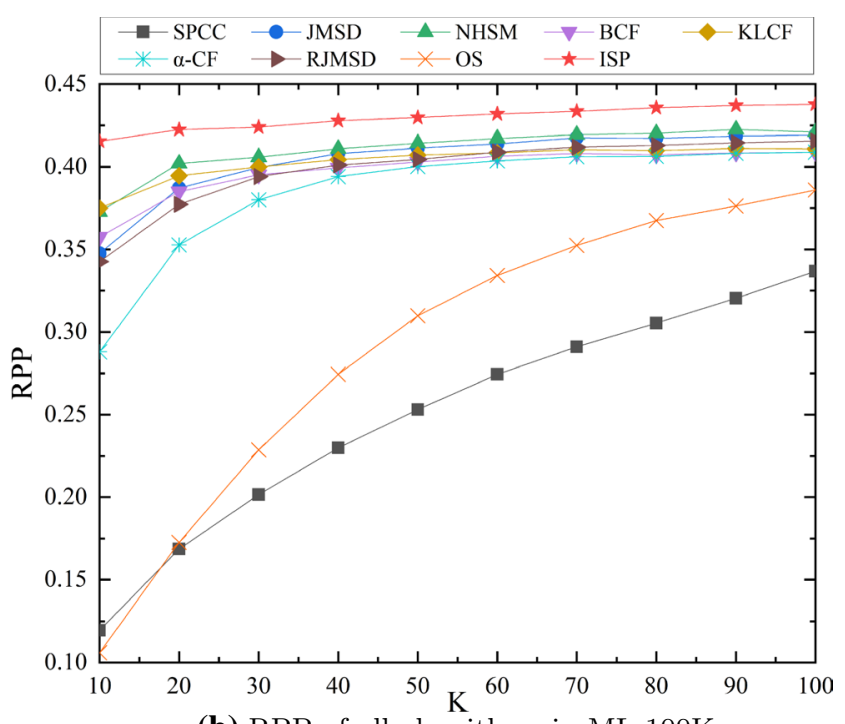

(b) RPP of all algorithms in ML-100K. 


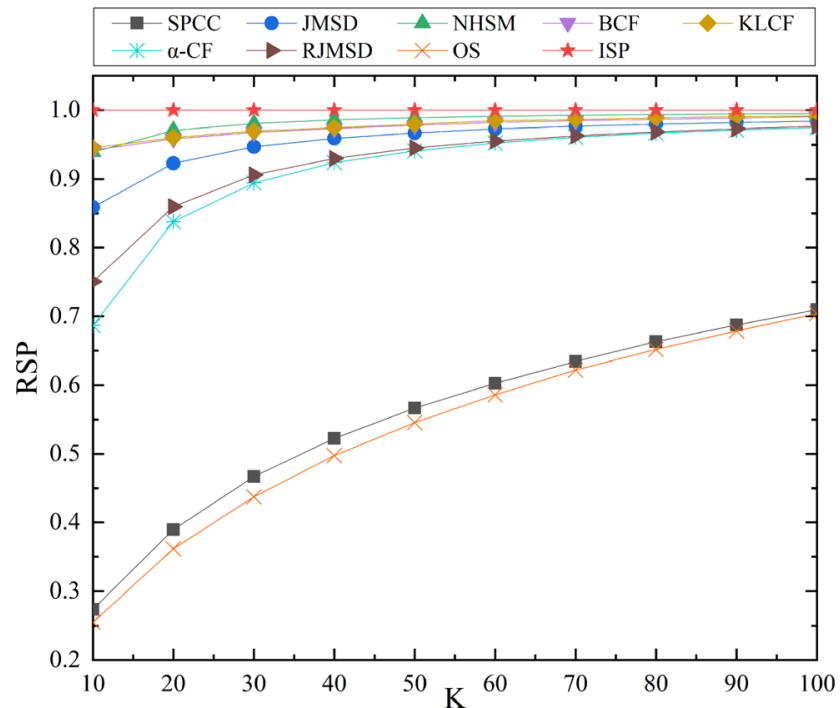

(a) RSP of all algorithms in ML-1M.

Fig. 8 RSP and RPP values of all algorithms in ML-1 M

Acknowledgements We thank the editors and the anonymous reviewers for their insightful comments which improved the quality of the paper.

Funding This work was supported by the National Natural Science Foundation of China (No. 61503124), the Science and Technology Department of Henan Province (No. 182102310935).

Availability of Data and Materials The data sets used during the current study are available in the MovieLens website by GroupLens team.

\section{Declarations}

Conflict of Interest The authors declare they have no conflicts of interest.

Ethics Approval and Consent to Participate: No applicable.

Consent for Publication: No applicable.

Author contributions THH carried out the related experiments and drafted the manuscript. ZWT contributed to analysis and manuscript preparation. WY participated in the design of the study and performed the statistical analysis. CZH and FTT conceived of the study, and participated in its design and coordination. All authors read and approved the final manuscript.

Open Access This article is licensed under a Creative Commons Attribution 4.0 International License, which permits use, sharing, adaptation, distribution and reproduction in any medium or format, as long as you give appropriate credit to the original author(s) and the source, provide a link to the Creative Commons licence, and indicate if changes were made. The images or other third party material in this article are included in the article's Creative Commons licence, unless indicated otherwise in a credit line to the material. If material is not included in the article's Creative Commons licence and your intended use is not permitted by statutory regulation or exceeds the permitted use, you will

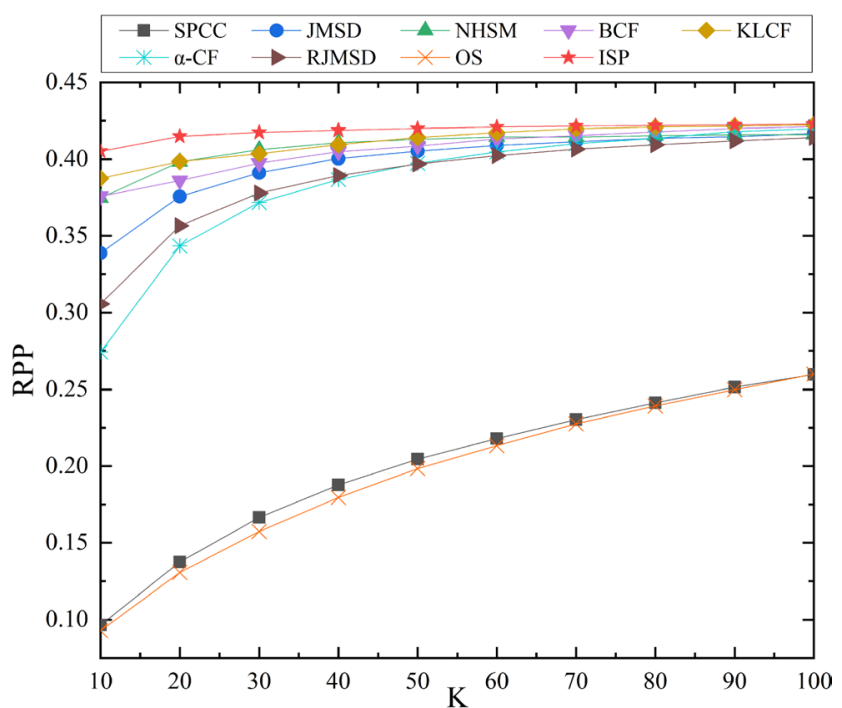

(b) RPP of all algorithms in ML-1M.

need to obtain permission directly from the copyright holder. To view a copy of this licence, visit http://creativecommons.org/licenses/by/4.0/.

\section{References}

1. Ahn, H.J.: A new similarity measure for collaborative filtering to alleviate the new user cold-starting problem. Inform. Sci. 178(1), 37-51 (2008)

2. Alhijawi, B., Al-Naymat, G., Obeid, N., Awajan, A.: Novel predictive model to improve the accuracy of collaborative filtering recommender systems. Inform. Syst. 96, 101670 (2021)

3. Bag, S., Kumar, S.K., Tiwari, M.K.: An efficient recommendation generation using relevant Jaccard similarity. Inform. Sci. 483, 53-64 (2019)

4. Bobadilla, J., Serradilla, F., Bernal, J.: A new collaborative filtering metric that improves the behavior of recommender systems. Knowl. Based Syst. 23(6), 520-528 (2010)

5. Boratto, L., Carta, S., Fenu, G.: Investigating the role of the rating prediction task in granularity-based group recommender systems and big data scenarios. Inform. Sci. 378, 424-443 (2017)

6. Camacho, L.A.G., Souza, S.N.A.: Social network data to alleviate cold-start in recommender system: a systematic review. Inform. Process. Manag. 54(4), 529-544 (2018)

7. Candillier, L., Meyer, F., Boullé, M.: Comparing state-of-the-art collaborative filtering systems. In: Machine Learning and Data Mining in Pattern Recognition, 5th International Conference, Leipzig, Germany, July 18-20, 2007, pp 548-562 (2007)

8. Chen, M.H., Teng, C.H., Chang, P.C.: Applying artificial immune systems to collaborative filtering for movie recommendation. Adv. Eng. Inform 29(4), 830-839 (2015)

9. Desrosiers, C., Karypis, G.: A comprehensive survey of neighborhood-based recommendation methods. In: Recommender Systems Handbook pp 107-144 (2011)

10. Ding, R., Liu, B., Gu, B., Li, J., Li, X.: Predictability of ensemble forecasting estimated using the Kullback-Leibler divergence in the lorenz model. Adv. Atmos. Sci. 36(8), 837-846 (2019) 
11. Feng, C., Liang, J., Song, P., Wang, Z.: A fusion collaborative filtering method for sparse data in recommender systems. Inform. Sci. 521, 365-379 (2020)

12. Gao, H., Li, B., Xie, W., Zhang, Y., Cai, K.: Csip: enhanced link prediction with context of social influence propagation. Big Data Res. 24, 100217 (2021)

13. Gazdar, A., Hidri, L.: A new similarity measure for collaborative filtering based recommender systems. Knowl. Based Syst. 188, 105058 (2020)

14. Geuens, S., Coussement, K., Bock, K.W.D.: A framework for configuring collaborative filtering-based recommendations derived from purchase data. Eur. J. Oper. Res. 265(1), 208-218 (2018)

15. Haifeng Liu, H.Z., Mian, A., Tian, H., Zhu, X.: A new user similarity model to improve the accuracy of collaborative filtering. Knowl. Based Syst. 56, 156-166 (2014)

16. Jamali, M., Ester, M.: TrustWalker: a random walk model for combining trust-based and item-based recommendation. In: Proceedings of the 15th ACM SIGKDD International Conference on Knowledge Discovery and Data Mining, Paris, France, June 28 - July 1, 2009, pp 397-406 (2009)

17. Khojamli, H., Razmara, J.: Survey of similarity functions on neighborhood-based collaborative filtering. Expert Syst. Appl. 185(6), 115482 (2021)

18. Kosub, S.: A note on the triangle inequality for the Jaccard distance. Pattern Recogn. Lett. 120, 36-38 (2019)

19. Kumar, P., Kumar, V., Thakur, R.S.: A new approach for rating prediction system using collaborative filtering. Iran J. Comput. Sci. 2, 81-87 (2019)

20. Nguyen, V., Sriboonchitta, S., Huynh, V.: Using community preference for overcoming sparsity and cold-start problems in collaborative filtering system offering soft ratings. Electron. Commer. Res. Appl. 26, 101-108 (2017)

21. Patra, B.K., Launonen, R., Ollikainen, V., Nandi, S.: A new similarity measure using Bhattacharyya coefficient for collaborative filtering in sparse data. Knowl. Based Syst. 82, 163-177 (2015)

22. Ponti, M., Kittler, J., Riva, M., de Campos, T.E., Zor, C.: A decision cognizant Kullback-Leibler divergence. Pattern Recogn. 61, 470-478 (2017)

23. Rodpysh, K.V., Mirabedini, S.J., Banirostam, T.: Resolving cold start and sparse data challenge in recommender systems using multi-level singular value decomposition. Comput. Elect. Eng. 94, $107361(2021)$

24. Sanchez, JL., Serradilla, F., Martinez, E., Bobadilla, J.: Choice of metrics used in collaborative filtering and their impact on recommender systems. In: 2008 2nd IEEE International Conference on Digital Ecosystems and Technologies, pp 432-436 (2008)

25. Sarwar, BM., Karypis, G., Konstan, JA., Riedl, J.: Item-based collaborative filtering recommendation algorithms. In: Proceedings of the Tenth International World Wide Web Conference, WWW 10, Hong Kong, China, May 1-5, 2001, pp 285-295 (2001)

26. Saxena, D., Lamest, M.: Information overload and coping strategies in the big data context: evidence from the hospitality sector. J. Inform. Sci. Princ. Pract. 44(3), 287-297 (2018)

27. Singh, P.K., Ahmed, R., Rajput, I.S., Choudhury: A comparative study on prediction approaches of item-based collaborative filtering in neighborhood-based recommendations. Wireless Personal Communications 121, 1-21 (2021)

28. Wang, Y., Deng, J., Gao, J., Zhang, P.: A hybrid user similarity model for collaborative filtering. Inform. Sci. 418-419, 102-118 (2017)

29. Wang, D., Yih, Y., Ventresca, M.: Improving neighbor-based collaborative filtering by using a hybrid similarity measurement. Expert Syst. Appl. 160, 113651 (2020)

30. Wang, Y., Wang, P., Liu, Z., Zhang, L.Y.: A new item similarity based on $\alpha$-divergence for collaborative filtering in sparse data. Expert Syst. Appl. 166, 114074 (2021)

31. Wei, J., He, J., Chen, K., Zhou, Y., Tang, Z.: Collaborative filtering and deep learning based recommendation system for cold start items. Expert Syst. Appl. 69, 29-39 (2017)

32. Wu, J., Chen, L., Feng, Y., Zheng, Z., Zhou, M., Wu, Z.: Predicting quality of service for selection by neighborhood-based collaborative filtering. IEEE Tran. Syst. Man Cybern. 43(2), 428-439 (2013)

33. Yang, Y., Hinde, C.J.: A new extension of fuzzy sets using rough sets: R-fuzzy sets. Inform. Sci. 180(3), 354-365 (2010)

34. Yang, J., Yao, Y.: A three-way decision based construction of shadowed sets from atanassov intuitionistic fuzzy sets. Inform. Sci. 577, 1-21 (2021)

35. Yu, S., Yang, M., Qu, Q., Shen, Y.: Contextual-boosted deep neural collaborative filtering model for interpretable recommendation. Expert Syst. Appl. 136, 365-375 (2019)

36. Zhang, J., Pu, P.: A recursive prediction algorithm for collaborative filtering recommender systems. In: Proceedings of the 2007 ACM Conference on Recommender Systems, pp 57-64 (2007)

37. Zhang, J., Peng, Q., Sun, S., Liu, C.: Collaborative filtering recommendation algorithm based on user preference derived from item domain features. Phys. A Stat. Mech. Appl. 396, 66-76 (2014)

38. Zhang, F., Qi, S., Liu, Q., Mao, M., Zeng, A.: Alleviating the data sparsity problem of recommender systems by clustering nodes in bipartite networks. Expert Syst. Appl. 149, 113346 (2020)

39. Zhang, L., Li, Z., Sun, X.: Iterative rating prediction for neighborhood-based collaborative filtering. Appl. Intell. 51(10), 6810 $6822(2021)$

Publisher's Note Springer Nature remains neutral with regard to jurisdictional claims in published maps and institutional affiliations. 